\title{
Detection Scheme for Space-time Block Codes Wireless Communications without Channel State Information
}

\author{
F. Delestre, E. Masoud, Y. Sun and A. Slaney \\ School of Electronic, Communication and Electrical Engineering \\ Faculty of Engineering and Information Sciences \\ University of Hertfordshire, Hatfield, Herts, AL10 9AB, UK
}

\begin{abstract}
In this paper, the new detection scheme proposed by Tarokh and Alamouti is investigated and expanded to two schemes of two transmit two receive antennas and four transmit one receive antennas. Previously, Tarokh and Alamouti, based on the simple transmit diversity scheme proposed by Alamouti, have provided two new detection methods to decode signals at the receiver without channel information. We expand their work to two and four transmit antennas with different number of receive antennas. We also demonstrate that the two methods are in fact the same with one being a special case of the other. The theory and detailed derivation of detection formulas for three cases, Tarokh and Alamouti work, two transmit two receive antennas and four transmit one receive antennas are presented.
\end{abstract}

\section{INTRODUCTION}

In the past few years, Multiple-Input Multiple-Output (MIMO) wireless communications have received much interest of research. Multiple antennas are employed at both the receiver and the transmitter in a MIMO communication system. Space-time block coding has emerged as one of the major techniques. Full transmit diversity is achieved with space-time block coding and the maximum likelihood decoding algorithm at the receiver for this technique is very simple.

Decoding space time codes requires channel state information (CSI). In practice, due to the changing environment, an estimation of the channel has to be made to extract the received signal. Orthogonal pilot sequences are often used for channel estimation in the literature [5].

The use of pilot sequences at the beginning of the transmission was exploited by Tarokh and Alamouti in [1] where the first transmitted symbols are the pilot then followed by the data in the next sequence. Indeed, in [1], a new detection scheme for transmit diversity was proposed. Two methods were presented, however formulations have not been given. The work was based on the simple transmit diversity technique proposed by Alamouti in [2], with only two transmit antennas and one receive antenna considered. The new scheme does not require channel state information to decode the received signal, which is an attractive feature.

The above has motivated us to consider the detection scheme further. Indeed, in this paper, we will, through detailed derivation of detection formulas, prove that the first new detection method proposed in [1] is actually a special case of

This research is funded by EPSRC (No.06001434) the second. In addition, Tarokh and Alamouti's work presented in [1] will be expanded to systems with two transmit and two receive antennas and to four transmit antennas with one receive antenna.

First, a review of the simple transmit diversity scheme proposed in [2] will be made. Then, the new detection method proposed by Tarokh and Alamouti will be derived thoroughly and next, it will be demonstrated that the first method of [1] is simply a special case of the second. This will be followed by the extension of the method to a MIMO scheme composed of two transmit and two receive antennas and to the four transmit antennas case. Conclusions will be drawn in the last section.

\section{A REVIEW OF ALAMOUTI'S SCHEME}

In this section, the method described in [2] is reviewed. Figure 1 shows a wireless communication systems with two transmit antennas and one receive antenna. Alamouti scheme contains three main operations: transmission, combination and detection.

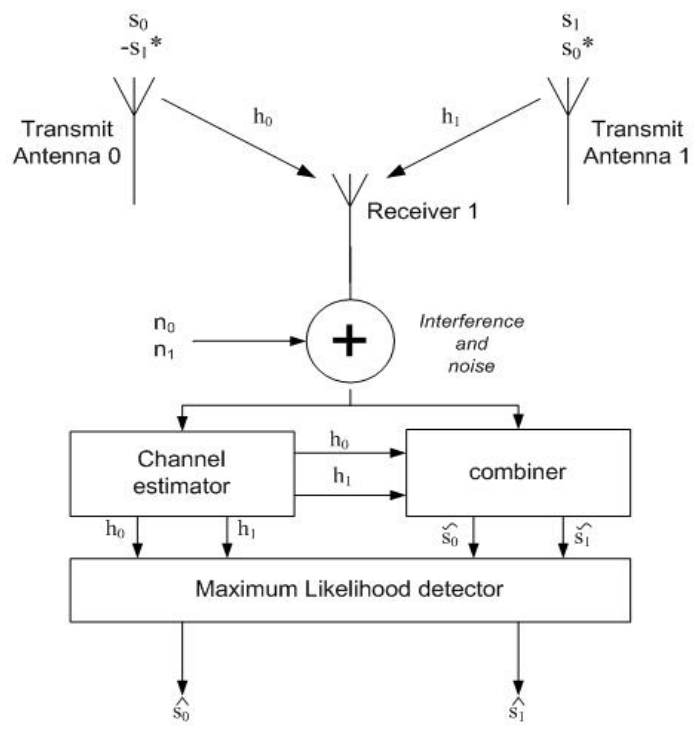

Figure 1. The two-branch transmit diversity scheme with one receiver [2] 
With the help of figure 1, it can be seen that at time t, two signals are sent from the transmitter at the same time. The two signals transmitted from antennas 0 and 1 are $\mathrm{s}_{0}$ and $\mathrm{s}_{1}$ respectively. Then at time $\mathrm{t}+\mathrm{T}$ where $\mathrm{T}$ is the symbol period, the signals $\left(-\mathrm{s}_{1} *\right)$ and $\left(\mathrm{s}_{0} *\right)$ are transmitted from antenna 0 and 1 respectively. These sequences are summarized in table I.

TABLE I: THE ENCODING AND TRANSMISSION SEQUENCE FOR THE TRANSMIT SYMBOLS

\begin{tabular}{|c|c|c|}
\hline Time Antenna & Tx 0 & $\mathrm{Tx} 1$ \\
\hline $\mathrm{t}$ & $\mathrm{s}_{0}$ & $\mathrm{~s}_{1}$ \\
\hline $\mathrm{t}+\mathrm{T}$ & $-\mathrm{s}_{1}{ }^{*}$ & $\mathrm{~s}_{0}{ }^{*}$ \\
\hline
\end{tabular}

Moreover, as it can be seen from figure 1, the transmission of each signal is through a channel described as $h_{0}$ and $h_{1}$ for antenna 0 and 1 respectively.

During transmission, noise is also added to the signal. Therefore, the received signals can be obtained as:

$$
\begin{aligned}
& r_{0}=r(t)=h_{0} s_{0}+h_{1} s_{1}+n_{0} \\
& r_{1}=r(t+T)=-h_{0} s_{1}^{*}+h_{1} s_{0}^{*}+n_{1}
\end{aligned}
$$

where $\mathrm{n}_{0}$ and $\mathrm{n}_{1}$ represent the complex noise and interference.

The combiner shown in figure 1 then combines the signals as:

$$
\begin{aligned}
& \widetilde{s_{0}}=h_{0}^{*} r_{0}+h_{1} r_{1}^{*} \\
& \widetilde{s_{1}}=h_{1}^{*} r_{0}-h_{0} r_{1}^{*}
\end{aligned}
$$

Substituting $r_{0}$ and $r_{1}$ in (1) into (2), we obtain:

$$
\begin{aligned}
& \widetilde{s_{0}}=\left(\left|h_{0}\right|^{2}+\left|h_{1}\right|^{2}\right) s_{0}+h_{0}^{*} n_{0}+h_{1} n_{1}^{*} \\
& \widetilde{s_{1}}=\left(\left|h_{0}\right|^{2}+\left|h_{1}\right|^{2}\right) s_{1}-h_{0} n_{1}^{*}+h_{1}^{*} n_{0}
\end{aligned}
$$

The two signals $\widetilde{s_{0}}$ and $\widetilde{s_{1}}$ coming from the combiner are then sent to the maximum likelihood detector. The detection rule for PSK signals is given in (4).

$$
\begin{array}{ll}
d^{2}\left(\widetilde{s_{0}}, s_{i}\right) \leq d^{2}\left(\widetilde{s_{0}}, s_{k}\right) & \forall i \neq k \\
d^{2}\left(\widetilde{s_{1}}, s_{i}\right) \leq d^{2}\left(\widetilde{s_{1}}, s_{k}\right) & \forall i \neq k
\end{array}
$$

where $\mathrm{d}^{2}(\mathrm{x}, \mathrm{y})=(\mathrm{x}-\mathrm{y})(\mathrm{x} * \mathrm{y} *)$

\section{THE NEW DETECTION METHOD AND DERIVATION}

As in [1], the assumption that the fade is constant over every 4 consecutive transmissions is made. Moreover, it is assumed that the signals $\mathrm{s}_{0}$ and $\mathrm{s}_{1}$ are known by the receiver as well as the received words $\mathrm{r}_{0}, \mathrm{r}_{1}, \mathrm{r}_{2}, \mathrm{r}_{3}, \mathrm{r}_{4}, \mathrm{r}_{5}$. Equations of the received words with transmitted signals $\mathrm{s}_{0}, \mathrm{~s}_{1}$ and $\mathrm{s}_{2}, \mathrm{~s}_{3}$ can be found in (5) and (6) respectively:

$$
\begin{aligned}
& r_{0}=r(t)=h_{0} s_{0}+h_{1} s_{1}+n_{0} \\
& r_{1}=r(t+T)=-h_{0} s_{1}^{*}+h_{1} s_{0}^{*}+n_{1} \\
& r_{2}=r(t+2 T)=h_{0} s_{2}+h_{1} s_{3}+n_{2} \\
& r_{3}=r(t+3 T)=-h_{0} s_{3}^{*}+h_{1} s_{2}^{*}+n_{3}
\end{aligned}
$$

The aim of this new detection method is to detect the two transmitted signals $s_{2}$ and $s_{3}$. Then, when those two signals are known, it will be possible to repeat the process in order to compute $s_{4}$ and $s_{5}$. The scheme needs only to know the two first transmitted symbols in order to compute the two following. With $\mathrm{s}_{4}$ and $\mathrm{s}_{5}$, it will be possible to find $\mathrm{s}_{6}$ and $\mathrm{s}_{7}$ and so on.

The first step for the receiver is the estimation of the channel $h_{0}$ and $h_{1}$. By writing (5) in the matrix format, it can be easily seen how estimation can be performed.

$$
\left[\begin{array}{l}
r_{0} \\
r_{1}
\end{array}\right]=\left[\begin{array}{cc}
s_{0} & s_{1} \\
-s_{1}^{*} & s_{0}^{*}
\end{array}\right]\left[\begin{array}{c}
h_{0} \\
h_{1}
\end{array}\right]+\left[\begin{array}{c}
n_{0} \\
n_{1}
\end{array}\right]
$$

Using (7) with the noise vector being dropped, we can obtain the following MMSE channel estimation.

$$
\left[\begin{array}{c}
\widetilde{h_{0}} \\
\widetilde{h_{1}}
\end{array}\right]=\frac{1}{\left|s_{0}\right|^{2}+\left|s_{1}\right|^{2}}\left[\begin{array}{cc}
s_{0}^{*} & -s_{1} \\
s_{1}^{*} & s_{0}
\end{array}\right]\left[\begin{array}{l}
r_{0} \\
r_{1}
\end{array}\right]
$$

Equation (8) can be written as

$$
\begin{aligned}
& \widetilde{h_{0}}=\frac{r_{0} s_{0}^{*}-r_{1} s_{1}}{\left|s_{0}\right|^{2}+\left|s_{1}\right|^{2}}=h_{0}+\frac{s_{0}^{*} n_{0}-s_{1} n_{1}}{\left|s_{0}\right|^{2}+\left|s_{1}\right|^{2}} \\
& \widetilde{h_{1}}=\frac{r_{0} s_{1}^{*}+r_{1} s_{0}}{\left|s_{0}\right|^{2}+\left|s_{1}\right|^{2}}=h_{1}+\frac{s_{1}^{*} n_{0}+s_{0} n_{1}}{\left|s_{0}\right|^{2}+\left|s_{1}\right|^{2}}
\end{aligned}
$$

Equation (9) was given without proof in [1] as Detection Method 2.

Using these estimations, it is possible to detect the two signals $s_{2}$ and $s_{3}$ by using (6). Once $s_{2}$ and $s_{3}$ are computed, equation (9) can be used to find $s_{4}$ and $s_{5}$ by using $s_{2}$ and $s_{3}$ as the pilot in place of $\mathrm{s}_{0}$ and $\mathrm{s}_{1}$ and by using the corresponding receive equations.

We now carry on with the following further derivations which are not directly available from [1].

Similar to (2), using (6), we can form the following combinations:

$$
\begin{aligned}
\widetilde{s_{2}} & =h_{0}^{*} r_{2}+h_{1} r_{3}^{*} \\
\widetilde{s_{3}} & =h_{1}^{*} r_{2}-h_{0} r_{3}^{*}
\end{aligned}
$$

Substitution of (9) into (10) leads to:

$$
\begin{aligned}
& \widetilde{s_{2}}=\frac{1}{\Delta_{s}}\left[s_{1}^{*}\left(r_{0} r_{3}^{*}-r_{1}^{*} r_{2}\right)+s_{0}\left(r_{0}^{*} r_{2}+r_{1} r_{3}^{*}\right)\right] \\
& \widetilde{s_{3}}=\frac{1}{\Delta_{s}}\left[s_{1}\left(r_{0}^{*} r_{2}+r_{1} r_{3}^{*}\right)-s_{0}^{*}\left(r_{0} r_{3}^{*}-r_{1}^{*} r_{2}\right)\right]
\end{aligned}
$$

where $\Delta_{\mathrm{s}}=\left|\mathrm{s}_{0}\right|^{2}+\left|\mathrm{s}_{1}\right|^{2}$.

Equation (11) can also be written as:

$$
\begin{aligned}
& \widetilde{s_{2}}=\frac{1}{\Delta_{s}}\left(A s_{1}^{*}+B s_{0}\right) \\
& \widetilde{s_{3}}=\frac{1}{\Delta_{s}}\left(B s_{1}-A s_{0}^{*}\right)
\end{aligned}
$$

Where $\mathrm{A}$ and $\mathrm{B}$ can be derived using (11), (5) and (6) as:

$$
\begin{aligned}
A & =r_{0} r_{3}^{*}-r_{1}^{*} r_{2} \\
& =\left(\left|h_{0}\right|^{2}+\left|h_{1}\right|^{2}\right)\left(s_{1} s_{2}-s_{0} s_{3}\right)+N_{1} \\
B & =r_{0}^{*} r_{2}+r_{1} r_{3}^{*} \\
& =\left(\left|h_{0}\right|^{2}+\left|h_{1}\right|^{2}\right)\left(s_{0}^{*} s_{2}+s_{1}^{*} s_{3}\right)+N_{2}
\end{aligned}
$$

where $\mathrm{N}_{1}$ and $\mathrm{N}_{2}$ are noise terms. 
It can be shown by substituting (13) into (12) that:

$$
\begin{aligned}
& \widetilde{s_{2}}=\left(\left|h_{0}\right|^{2}+\left|h_{1}\right|^{2}\right) s_{2}+N_{3} \\
& \widetilde{s_{3}}=\left(\left|h_{0}\right|^{2}+\left|h_{1}\right|^{2}\right) s_{3}+N_{4}
\end{aligned}
$$

where $\mathrm{N}_{3}$ and $\mathrm{N}_{4}$ are noise terms.

From the above; detailed derivation of the general formulae of the new detection scheme presented in [1], we now consider a special case of $\Delta_{\mathrm{s}}=\left|\mathrm{s}_{0}\right|^{2}+\left|\mathrm{s}_{1}\right|^{2}=1$ which means $\left|\mathrm{s}_{0}\right|^{2}=\left|\mathrm{s}_{1}\right|^{2}=1 / 2$ for PSK. In this case, equation (12) becomes:

$$
\begin{gathered}
\widetilde{s_{2}}=A s_{1}^{*}+B s_{0} \\
\widetilde{s_{3}}=B s_{1}-A s_{0}^{*}
\end{gathered}
$$

Equations (13), (14) and (15) constitute Detection Method 1 proposed by Tarokh and Alamouti in [1], with the same assumption that $\left|s_{0}\right|^{2}=\left|s_{1}\right|^{2}=1 / 2$. Therefore, we have demonstrated that this method is not a different method, but a special case of the second method.

\section{DETECTION SCHEME FOR TWO TRANSMIT AND TWO RECEIVE ANTENNA SYSTEMS}

In the following, the detection scheme is extended for MIMO wireless systems with two transmit antennas and two receive antennas. First, the pilot is sent in order to estimate the channel. This will result in four equations at the receiver, one at time $\mathrm{t}$ and one at $\mathrm{t}+\mathrm{T}$ for each receive antenna. Then the signals $s_{2}$ and $s_{3}$ are sent.

At receive antenna one, equation (16) can be written for known $\mathrm{s}_{0}$ and $\mathrm{s}_{1}$ :

$$
\begin{aligned}
& r_{0}=r(t)=h_{0} s_{0}+h_{1} s_{1}+n_{0} \\
& r_{1}=r(t+T)=-h_{0} s_{1}^{*}+h_{1} s_{0}^{*}+n_{1}
\end{aligned}
$$

And for transmitted symbols $s_{2}$ and $s_{3}$, we can write:

$$
\begin{aligned}
& r_{2}=r(t+2 T)=h_{0} s_{2}+h_{1} s_{3}+n_{2} \\
& r_{3}=r(t+3 T)=-h_{0} s_{3}^{*}+h_{1} s_{2}^{*}+n_{3}
\end{aligned}
$$

For receive antenna two, we can write another four equations similarly. At time $\mathrm{t}$ and $\mathrm{t}+\mathrm{T}$, the received symbols are as follows:

$$
\begin{aligned}
& r_{4}=r(t)=h_{2} s_{0}+h_{3} s_{1}+n_{4} \\
& r_{5}=r(t+T)=-h_{2} s_{1}^{*}+h_{3} s_{0}^{*}+n_{5}
\end{aligned}
$$

And for the transmitted symbols $s_{2}$ and $s_{3}$, we have:

$$
\begin{aligned}
& r_{6}=r(t+2 T)=h_{2} s_{2}+h_{3} s_{3}+n_{6} \\
& r_{7}=r(t+3 T)=-h_{2} s_{3}^{*}+h_{3} s_{2}^{*}+n_{7}
\end{aligned}
$$

The channels can be estimated from (16) and (18). As it has been done in Section III, equation (16) can be written in matrix form as:

$$
\left[\begin{array}{l}
r_{0} \\
r_{1}
\end{array}\right]=\left[\begin{array}{cc}
s_{0} & s_{1} \\
-s_{1}^{*} & s_{0}^{*}
\end{array}\right]\left[\begin{array}{l}
h_{0} \\
h_{1}
\end{array}\right]+\left[\begin{array}{l}
n_{0} \\
n_{1}
\end{array}\right]
$$

and for (18):

$$
\left[\begin{array}{l}
r_{4} \\
r_{5}
\end{array}\right]=\left[\begin{array}{cc}
s_{0} & s_{1} \\
-s_{1}^{*} & s_{0}^{*}
\end{array}\right]\left[\begin{array}{l}
h_{2} \\
h_{3}
\end{array}\right]+\left[\begin{array}{l}
n_{4} \\
n_{5}
\end{array}\right]
$$

In the same process as in Section III, with (20) and (21), the equations of channel estimations can be derived as given in (22) and (23) respectively.

$$
\begin{aligned}
& \widetilde{h_{0}}=\frac{r_{0} s_{0}^{*}-r_{1} s_{1}}{\left|s_{0}\right|^{2}+\left|s_{1}\right|^{2}}=h_{0}+\frac{s_{0}^{*} n_{0}-s_{1} n_{1}}{\left|s_{0}\right|^{2}+\left|s_{1}\right|^{2}} \\
& \widetilde{h_{1}}=\frac{r_{0} s_{1}^{*}+r_{1} s_{0}}{\left|s_{0}\right|^{2}+\left|s_{1}\right|^{2}}=h_{1}+\frac{s_{1}^{*} n_{0}+s_{0} n_{1}}{\left|s_{0}\right|^{2}+\left|s_{1}\right|^{2}} \\
& \widetilde{h_{2}}=\frac{r_{4} s_{0}^{*}-r_{5} s_{1}}{\left|s_{0}\right|^{2}+\left|s_{1}\right|^{2}}=h_{2}+\frac{s_{0}^{*} n_{4}-s_{1} n_{5}}{\left|s_{0}\right|^{2}+\left|s_{1}\right|^{2}} \\
& \widetilde{h_{3}}=\frac{r_{4} s_{1}^{*}+r_{5} s_{0}}{\left|s_{0}\right|^{2}+\left|s_{1}\right|^{2}}=h_{3}+\frac{s_{1}^{*} n_{4}+s_{0} n_{5}}{\left|s_{0}\right|^{2}+\left|s_{1}\right|^{2}}
\end{aligned}
$$

From [2], the formulas for estimation of signals $s_{2}$ and $s_{3}$ can be written based on (17) and (19) as

$$
\begin{aligned}
& \widetilde{s_{2}}=h_{0}^{*} r_{2}+h_{1} r_{3}^{*}+h_{2}^{*} r_{6}+h_{3} r_{7}^{*} \\
& \widetilde{s_{3}}=h_{1}^{*} r_{2}-h_{0} r_{3}^{*}+h_{3}^{*} r_{6}-h_{2} r_{7}^{*}
\end{aligned}
$$

By replacing $h_{0}, h_{1}, h_{2}, h_{3}$ in (24) by the corresponding expressions in (22) and (23), it can be derived:

$$
\begin{aligned}
\widetilde{s_{2}}= & \frac{1}{\Delta_{s}}\left[s_{1}^{*}\left(r_{0} r_{3}^{*}-r_{1}^{*} r_{2}+r_{4} r_{7}^{*}-r_{5}^{*} r_{6}\right)+\right. \\
& \left.s_{0}\left(r_{0}^{*} r_{2}+r_{1} r_{3}^{*}+r_{4}^{*} r_{6}+r_{5} r_{7}^{*}\right)\right] \\
\widetilde{s_{3}}= & \frac{1}{\Delta_{s}}\left[s_{1}\left(r_{0}^{*} r_{2}+r_{1} r_{3}^{*}+r_{4}^{*} r_{6}+r_{5} r_{7}^{*}\right)-\right. \\
& \left.s_{0}^{*}\left(r_{0} r_{3}^{*}-r_{1}^{*} r_{2}+r_{4} r_{7}^{*}-r_{5}^{*} r_{6}\right)\right]
\end{aligned}
$$

Where $\Delta_{\mathrm{s}}=\left|\mathrm{s}_{0}\right|^{2}+\left|\mathrm{s}_{1}\right|^{2}$.

Equation (25) can also be written as:

$$
\begin{aligned}
& \widetilde{s_{2}}=\frac{1}{\Delta_{s}}\left(A s_{1}^{*}+B s_{0}\right) \\
& \widetilde{s_{3}}=\frac{1}{\Delta_{s}}\left(B s_{1}-A s_{0}^{*}\right)
\end{aligned}
$$

Where A and B can be obtained from (16), (17), (18), (19) and (25) as:

$$
\begin{aligned}
A & =r_{0} r_{3}^{*}-r_{1}^{*} r_{2}+r_{4} r_{7}^{*}-r_{5}^{*} r_{6} \\
& =\Delta_{\mathrm{h}}\left(s_{1} s_{2}-s_{0} s_{3}\right)+N_{1} \\
B & =r_{0}^{*} r_{2}+r_{1} r_{3}^{*}+r_{4}^{*} r_{6}+r_{5} r_{7}^{*} \\
& =\Delta_{\mathrm{h}}\left(s_{0}^{*} s_{2}+s_{1}^{*} s_{3}\right)+N_{2}
\end{aligned}
$$

where $\Delta_{\mathrm{h}}=\left|\mathrm{h}_{0}\right|^{2}+\left|\mathrm{h}_{1}\right|^{2}+\left|\mathrm{h}_{2}\right|^{2}+\left|\mathrm{h}_{3}\right|^{2}$ and $\mathrm{N}_{1}, \mathrm{~N}_{2}$ are the noisy terms.

Substituting (27) into (26) yields:

$$
\begin{gathered}
\widetilde{s_{2}}=\Delta_{\mathrm{h}} s_{2}+N_{3} \\
\widetilde{s_{3}}=\Delta_{\mathrm{h}} s_{3}+N_{4}
\end{gathered}
$$

where $\mathrm{N}_{3}$ and $\mathrm{N}_{4}$ are noise terms. 
Assuming that the constellation points have equal energy normalized to $1 / 2$, it can be deduced that the value of $\Delta_{\mathrm{s}}$ is equal to 1 . Thus, equation (26) can be simplified as:

$$
\begin{aligned}
\widetilde{s_{2}} & =A s_{1}^{*}+B s_{0} \\
\widetilde{s_{3}} & =B s_{1}-A s_{0}^{*}
\end{aligned}
$$

The above special case can be used for PSK modulations. In the above, the detailed results have been derived for two receive antennas. Following the same procedure, the detection method can also be formulated for more receive antennas.

\section{DETECTION SCHEME FOR FOUR TRANSMIT ANTENNAS AND ONE RECEIVE ANTENNA}

In this section, the method of detection for four transmit antennas and one receive antenna is presented. The half-rate complex $\mathrm{G}_{4}$ code [6] is used. The fading channels are assumed to be constant over 16 symbol periods. In this cases, the transmitted pilot symbols $\mathrm{s}_{0}, \mathrm{~s}_{1}, \mathrm{~s}_{2}, \mathrm{~s}_{3}$ and received word $\mathrm{r}_{0}$, $\mathrm{r}_{1}, \ldots, \mathrm{r}_{7}$ are known at the receiver. The received signals can be found in (30).

$$
\begin{aligned}
& r_{0}=r(t)=h_{0} s_{0}+h_{1} s_{1}+h_{2} s_{2}+h_{3} s_{3}+n_{0} \\
& r_{1}=r(t+T)=-h_{0} s_{1}+h_{1} s_{0}-h_{2} s_{3}+h_{3} s_{2}+n_{1} \\
& r_{2}=r(t+2 T)=-h_{0} s_{2}+h_{1} s_{3}+h_{2} s_{0}-h_{3} s_{1}+n_{2} \\
& r_{3}=r(t+3 T)=-h_{0} s_{3}-h_{1} s_{2}+h_{2} s_{1}+h_{3} s_{0}+n_{3} \\
& r_{4}=r(t+4 T)=h_{0} s_{0}^{*}+h_{1} s_{1}^{*}+h_{2} s_{2}^{*}+h_{3} s_{3}^{*}+n_{4} \\
& r_{5}=r(t+5 T)=-h_{0} s_{1}^{*}+h_{1} s_{0}^{*}-h_{2} s_{3}^{*}+h_{3} s_{2}^{*}+n_{5} \\
& r_{6}=r(t+6 T)=-h_{0} s_{2}^{*}+h_{1} s_{3}^{*}+h_{2} s_{0}^{*}-h_{3} s_{1}^{*}+n_{6} \\
& r_{7}=r(t+7 T)=-h_{0} s_{3}^{*}-h_{1} s_{2}^{*}+h_{2} s_{1}^{*}+h_{3} s_{0}^{*}+n_{7}
\end{aligned}
$$

The received equations for the transmitted symbols $\mathrm{s}_{4}, \mathrm{~s}_{5}$, $\mathrm{s}_{6}$ and $\mathrm{s}_{7}$ can be written as in (31).

$$
\begin{aligned}
& r_{8}=r(t+8 T)=h_{0} s_{4}+h_{1} s_{5}+h_{2} s_{6}+h_{3} s_{7}+n_{8} \\
& r_{9}=r(t+9 T)=-h_{0} s_{5}+h_{1} s_{4}-h_{2} s_{7}+h_{3} s_{6}+n_{9} \\
& r_{10}=r(t+10 T)=-h_{0} s_{6}+h_{1} s_{7}+h_{2} s_{4}-h_{3} s_{5}+n_{10} \\
& r_{11}=r(t+11 T)=-h_{0} s_{7}-h_{1} s_{6}+h_{2} s_{5}+h_{3} s_{4}+n_{11} \\
& r_{12}=r(t+12 T)=h_{0} s_{4}^{*}+h_{1} s_{5}^{*}+h_{2} s_{6}^{*}+h_{3} s_{7}^{*}+n_{12} \\
& r_{13}=r(t+13 T)=-h_{0} s_{5}^{*}+h_{1} s_{4}^{*}-h_{2} s_{7}^{*}+h_{3} s_{6}^{*}+n_{13} \\
& r_{14}=r(t+14 T)=-h_{0} s_{6}^{*}+h_{1} s_{7}^{*}+h_{2} s_{4}^{*}-h_{3} s_{5}^{*}+n_{14} \\
& r_{15}=r(t+15 T)=-h_{0} s_{7}^{*}-h_{1} s_{6}^{*}+h_{2} s_{5}^{*}+h_{3} s_{4}^{*}+n_{15}
\end{aligned}
$$

Where $\mathrm{r}_{8}, \mathrm{r}_{9} \ldots, \mathrm{r}_{15}$ are also known at the receiver.

Equations (30) can be written in the matrix format in (32):

$$
\left[\begin{array}{l}
r_{0} \\
r_{1} \\
r_{2} \\
r_{3} \\
r_{4} \\
r_{5} \\
r_{6} \\
r_{7}
\end{array}\right]=\left[\begin{array}{cccc}
s_{0} & s_{1} & s_{2} & s_{3} \\
-s_{1} & s_{0} & -s_{3} & s_{2} \\
-s_{2} & s_{3} & s_{0} & -s_{1} \\
-s_{3} & -s_{2} & s_{1} & s_{0} \\
s_{0}^{*} & s_{1}^{*} & s_{2}^{*} & s_{3}^{*} \\
-s_{1}^{*} & s_{0}^{*} & -s_{3}^{*} & s_{2}^{*} \\
-s_{2}^{*} & s_{3}^{*} & s_{0}^{*} & -s_{1}^{*} \\
-s_{3}^{*} & -s_{2}^{*} & s_{1}^{*} & s_{0}^{*}
\end{array}\right]\left[\begin{array}{l}
h_{0} \\
h_{1} \\
h_{2} \\
h_{3}
\end{array}\right]+\left[\begin{array}{l}
n_{0} \\
n_{1} \\
n_{2} \\
n_{3}
\end{array}\right]
$$

The equations for the channel estimation can be derived from (32) as:

$$
\begin{aligned}
& \widetilde{h_{0}}=\frac{r_{0} s_{0}^{*}-r_{1} s_{1}^{*}-r_{2} s_{2}^{*}-r_{3} s_{3}^{*}+r_{4} s_{0}-r_{5} s_{1}-r_{6} s_{2}-r_{7} s_{3}}{2\left(\left|s_{0}\right|^{2}+\left|s_{1}\right|^{2}+\left|s_{2}\right|^{2}+\left|s_{3}\right|^{2}\right)} \\
& \widetilde{h_{1}}=\frac{r_{0} s_{1}^{*}+r_{1} s_{0}^{*}+r_{2} s_{3}^{*}-r_{3} s_{2}^{*}+r_{4} s_{1}+r_{5} s_{0}+r_{6} s_{3}-r_{7} s_{2}}{2\left(\left|s_{0}\right|^{2}+\left|s_{1}\right|^{2}+\left|s_{2}\right|^{2}+\left|s_{3}\right|^{2}\right)} \\
& \widetilde{h_{2}}=\frac{r_{0} s_{2}^{*}-r_{1} s_{3}^{*}+r_{2} s_{0}^{*}+r_{3} s_{1}^{*}+r_{4} s_{2}-r_{5} s_{3}+r_{6} s_{0}+r_{7} s_{1}}{2\left(\left|s_{0}\right|^{2}+\left|s_{1}\right|^{2}+\left|s_{2}\right|^{2}+\left|s_{3}\right|^{2}\right)} \\
& \widetilde{h_{3}}=\frac{r_{0} s_{3}^{*}+r_{1} s_{2}^{*}-r_{2} s_{1}^{*}+r_{3} s_{0}^{*}+r_{4} s_{3}+r_{5} s_{2}-r_{6} s_{1}+r_{7} s_{0}}{2\left(\left|s_{0}\right|^{2}+\left|s_{1}\right|^{2}+\left|s_{2}\right|^{2}+\left|s_{3}\right|^{2}\right)}
\end{aligned}
$$

The formulas for the combined signals can be written based on (31) as [7].

$$
\begin{gathered}
\widetilde{s_{4}}=h_{0}^{*} r_{8}+h_{1}^{*} r_{9}+h_{2}^{*} r_{10}+h_{3}^{*} r_{11}+ \\
h_{0} r_{12}^{*}+h_{1} r_{13}^{*}+h_{2} r_{14}^{*}+h_{3} r_{15}^{*} \\
\widetilde{s_{5}}=h_{1}^{*} r_{8}-h_{0}^{*} r_{9}-h_{3}^{*} r_{10}+h_{2}^{*} r_{11}+ \\
h_{1} r_{12}^{*}-h_{0} r_{13}^{*}-h_{3} r_{14}^{*}+h_{2} r_{15}^{*} \\
\widetilde{s_{6}}=h_{2}^{*} r_{8}+h_{3}^{*} r_{9}-h_{0}^{*} r_{10}-h_{1}^{*} r_{11}+ \\
h_{2} r_{12}^{*}+h_{3} r_{13}^{*}-h_{0} r_{14}^{*}-h_{1} r_{15}^{*} \\
\widetilde{s_{7}}=h_{3}^{*} r_{8}-h_{2}^{*} r_{9}+h_{1}^{*} r_{10}-h_{0}^{*} r_{11}+ \\
h_{3} r_{12}^{*}-h_{2} r_{13}^{*}+h_{1} r_{14}^{*}-h_{0} r_{15}^{*}
\end{gathered}
$$

Replacing the channels by their estimated expressions obtained in (33) and therefore (34) becomes:

$$
\begin{aligned}
& \widetilde{s_{4}}=\frac{1}{2 \Delta_{s}}\left(A s_{0}+B s_{1}+C s_{2}+D s_{3}+E s_{0}^{*}+F s_{1}^{*}+G s_{2}^{*}+H s_{3}^{*}\right) \\
& \widetilde{s_{5}}=\frac{1}{2 \Delta_{s}}\left(-B s_{0}+A s_{1}+D s_{2}-C s_{3}-F s_{0}^{*}+E s_{1}^{*}+H s_{2}^{*}-G s_{3}^{*}\right) \\
& \widetilde{s_{6}}=\frac{1}{2 \Delta_{s}}\left(-C s_{0}-D s_{1}+A s_{2}+B s_{3}-G s_{0}^{*}-H s_{1}^{*}+E s_{2}^{*}+F s_{3}^{*}\right) \\
& \widetilde{s_{7}}=\frac{1}{2 \Delta_{s}}\left(-D s_{0}+C s_{1}-B s_{2}+A s_{3}-H s_{0}^{*}+G s_{1}^{*}-F s_{2}^{*}+E s_{3}^{*}\right)
\end{aligned}
$$

where $\Delta_{\mathrm{s}}=\left|\mathrm{s}_{0}\right|^{2}+\left|\mathrm{s}_{1}\right|^{2}+\left|\mathrm{s}_{2}\right|^{2}+\left|\mathrm{s}_{3}\right|^{2}$ and A, B, C, D, E, F, G and H are given in (36).

$$
\begin{aligned}
& A=r_{0}^{*} r_{8}+r_{1}^{*} r_{9}+r_{2}^{*} r_{10}+r_{3}^{*} r_{11}+r_{4} r_{12}^{*}+r_{5} r_{13}^{*}+r_{6} r_{14}^{*}+r_{7} r_{15}^{*} \\
& =2 \Delta_{\mathrm{h}}\left(s_{0}^{*} s_{4}+s_{1}^{*} s_{5}+s_{2}^{*} s_{6}+s_{3}^{*} s_{7}\right)+N_{1} \\
& B=-r_{1}^{*} r_{8}+r_{0}^{*} r_{9}+r_{3}^{*} r_{10}-r_{2}^{*} r_{11}-r_{5} r_{12}^{*}+r_{4} r_{13}^{*}+r_{7} r_{14}^{*}-r_{6} r_{15}^{*} \\
& =2 \Delta_{\mathrm{h}}\left(s_{1}^{*} s_{4}-s_{0}^{*} s_{5}+s_{3}^{*} s_{6}-s_{2}^{*} s_{7}\right)+N_{2} \\
& C=-r_{2}^{*} r_{8}-r_{3}^{*} r_{9}+r_{0}^{*} r_{10}+r_{1}^{*} r_{11}-r_{6} r_{12}^{*}-r_{7} r_{13}^{*}+r_{4} r_{14}^{*}-r_{5} r_{15}^{*} \\
& =2 \Delta_{\mathrm{h}}\left(s_{2}^{*} s_{4}-s_{3}^{*} s_{5}-s_{0}^{*} s_{6}+s_{1}^{*} s_{7}\right)+N_{3} \\
& D=-r_{3}^{*} r_{8}+r_{2}^{*} r_{9}-r_{1}^{*} r_{10}+r_{0}^{*} r_{11}-r_{7} r_{12}^{*}+r_{6} r_{13}^{*}-r_{5} r_{14}^{*}+r_{4} r_{15}^{*} \\
& =2 \Delta_{\mathrm{h}}\left(s_{3}^{*} s_{4}+s_{2}^{*} s_{5}-s_{1}^{*} s_{6}-s_{0}^{*} s_{7}\right)+N_{4} \\
& E=r_{4}^{*} r_{8}+r_{5}^{*} r_{9}+r_{6}^{*} r_{10}+r_{7}^{*} r_{11}+r_{0} r_{12}^{*}+r_{1} r_{13}^{*}+r_{2} r_{14}^{*}+r_{3} r_{15}^{*} \\
& =2 \Delta_{\mathrm{h}}\left(s_{0} s_{4}+s_{1} s_{5}+s_{2} s_{6}+s_{3} s_{7}\right)+N_{5} \\
& F=-r_{5}^{*} r_{8}+r_{4}^{*} r_{9}+r_{7}^{*} r_{10}-r_{6}^{*} r_{11}-r_{1} r_{12}^{*}+r_{0} r_{13}^{*}+r_{3} r_{14}^{*}-r_{2} r_{15}^{*} \\
& =2 \Delta_{\mathrm{h}}\left(s_{1} s_{4}-s_{0} s_{5}+s_{3} s_{6}-s_{2} s_{7}\right)+N_{6} \\
& G=-r_{6}^{*} r_{8}-r_{7}^{*} r_{9}+r_{4}^{*} r_{10}+r_{5}^{*} r_{11}-r_{2} r_{12}^{*}-r_{3} r_{13}^{*}+r_{0} r_{14}^{*}+r_{1} r_{15}^{*} \\
& =2 \Delta_{\mathrm{h}}\left(s_{2} s_{4}-s_{3} s_{5}-s_{0} s_{6}+s_{1} s_{7}\right)+N_{7} \\
& H=-r_{7}^{*} r_{8}+r_{6}^{*} r_{9}-r_{5}^{*} r_{10}+r_{4}^{*} r_{11}-r_{3} r_{12}^{*}+r_{2} r_{13}^{*}-r_{1} r_{14}^{*}+r_{0} r_{15}^{*} \\
& =2 \Delta_{\mathrm{h}}\left(s_{3} s_{4}+s_{2} s_{5}-s_{1} s_{6}-s_{0} s_{7}\right)+N_{8}
\end{aligned}
$$

Where $\Delta_{\mathrm{h}}=\left|\mathrm{h}_{0}\right|^{2}+\left|\mathrm{h}_{1}\right|^{2}+\left|\mathrm{h}_{2}\right|^{2}+\left|\mathrm{h}_{3}\right|^{2}$ and $\mathrm{N}_{1}, \ldots, \mathrm{N}_{8}$ are the noisy terms. 
Substituting (36) into (35), the final equations for the signal detections can be derived as (37):

$$
\begin{gathered}
\widetilde{s_{4}}=\Delta_{\mathrm{h}} s_{4}+N_{9} \\
\widetilde{s_{5}}=\Delta_{\mathrm{h}} s_{5}+N_{10} \\
\widetilde{s_{6}}=\Delta_{\mathrm{h}} s_{6}+N_{11} \\
\widetilde{s_{7}}=\Delta_{\mathrm{h}} s_{7}+N_{12}
\end{gathered}
$$

where $\mathrm{N}_{9}, \mathrm{~N}_{10}, \mathrm{~N}_{11}$ and $\mathrm{N}_{12}$ are the noisy terms.

Following the same procedure as used for two transmit antennas and two receive antennas, the derivations for the channel estimation and signal detection can also be conducted for four transmit antennas and more receive antennas.

\section{RESULTS}

In the following, simulation results are provided for the detection methods proposed in this work. Results for two and four transmit antennas with one; two, three and four receive antennas are presented. All simulations were realized in the same condition, using Rayleigh fading and QPSK modulation.

Figure 2 shows the simulation results for two transmit antennas with known CSI and using the detection method proposed in this paper (no CSI). The simulation results for four transmit antennas with and without CSI are given in fig 3 . The results in both figures show a $3 \mathrm{~dB}$ loss of the detection method compared with the ideal channel case.

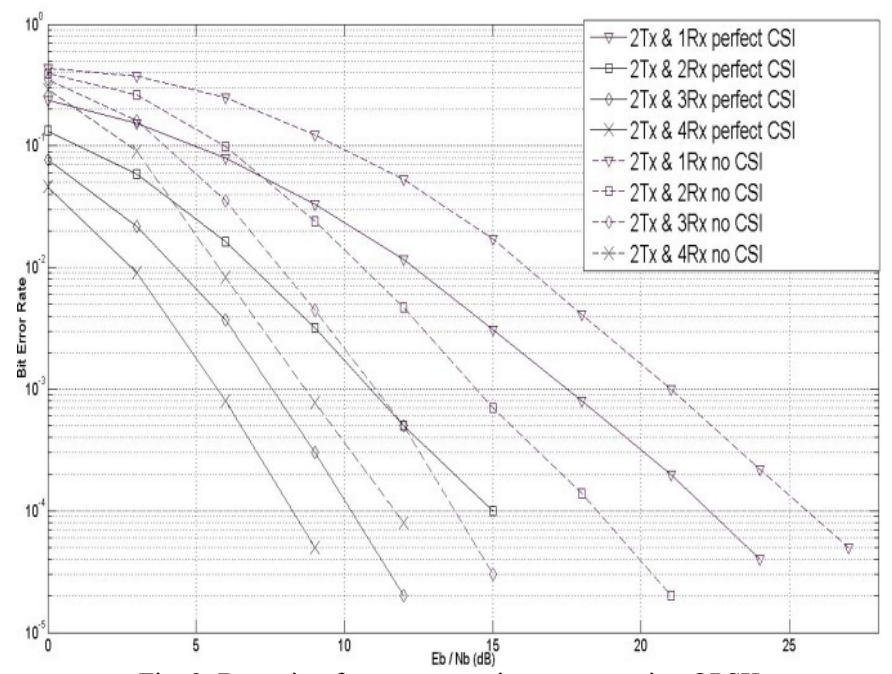

Fig. 2. Detection for two transmit antennas using QPSK

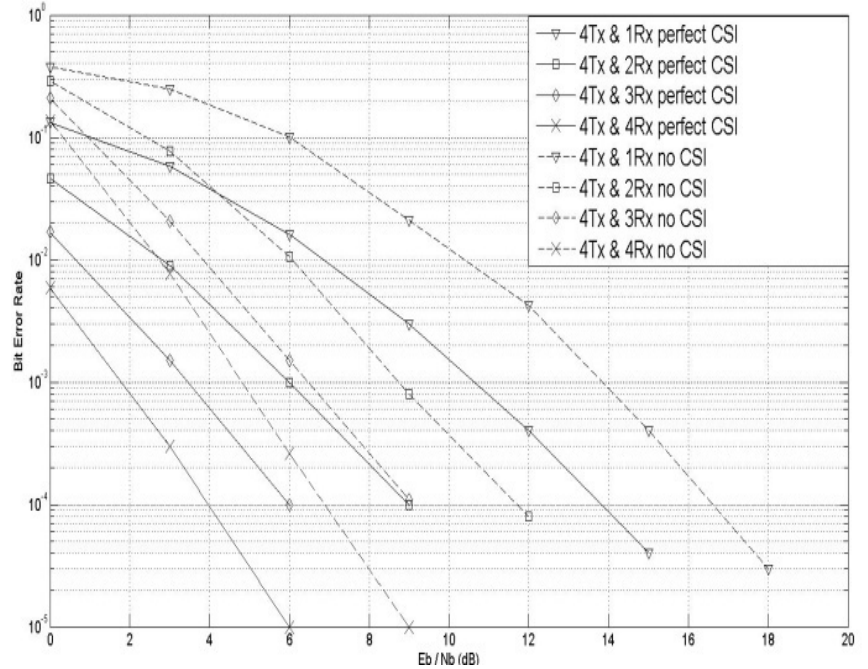

Fig. 3. Detection for four transmit antennas using QPSK

\section{CONCLUSIONS}

In this paper, we have further studied the detection scheme proposed by Tarokh and Alamouti in [1]. A comprehensive derivation of detection formulas has been conducted and we have demonstrated that the two detection methods are actually the same, with one being a special case only. In this paper, we have also extended Tarokh and Alamouti's detection scheme to wireless systems using two transmit antennas and four transmit antennas for up to four receive antennas. Detailed derivations and simulations results of these extensions have been given.

\section{REFERENCES}

[1] V. Tarokh, S.M. Alamouti and P. Poon, "New detection schemes for transmit diversity with no channel estimation", Proc. IEEE International Conference on Universal Personal Communications, Vol.2, pp.917-920, Florence, October 1998.

[2] S.M. Alamouti, "A simple transmit diversity technique for wireless communications", IEEE Journal on Selected Areas in Communications, Vol.16, No.8, pp.1451-1458, October 1998.

[3] E. Masoud and Y. Sun, "Joint channel estimation and data detection for space-time block coding with no channel state information", IET Seminar on Smart Antennas and Cooperative Communication, London, October 2007.

[4] A. Slaney and Y. Sun "Space-time coding for wireless communications: an overview", IEE Proceedings: Communications, Vol. 153, No.4, pp.509-518, August 2006.

[5] J. Yang, Y. Sun, J.M. Senior and N. Pem, "Channel estimation for wireless communications using space-time block coding techniques", IEEE International Symposium on Circuits and Systems, Japan, May 2003.

[6] V. Tarokh, H. Jafarkhani and A.R. Calderbank, "Space-time block codes from orthogonal designs", IEEE Transactions on Information Theory, Vol.45, No.5, pp.1456-1467, July 1999.

[7] V.Tarokh, H. Jafarkhani and A.R. Calderbank, "Space-time block coding for wireless communications: performance results", IEEE Journal on Selected Areas in Communication, Vol.17, No.3, pp.451-460, March 1999. 